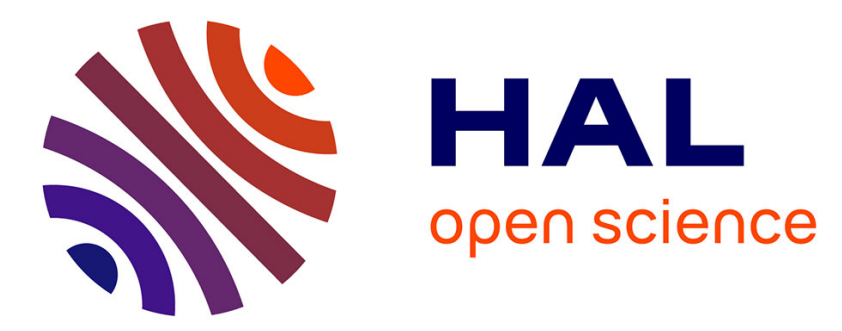

\title{
Impact de produits phytopharmaceutiques sur les microarthropodes du sol en culture de maïs irrigué: approche fonctionnelle par la méthode des sacs de litière \\ Jérôme Cortet, N. Poinsot-Balaguer
}

\section{- To cite this version:}

Jérôme Cortet, N. Poinsot-Balaguer. Impact de produits phytopharmaceutiques sur les microarthropodes du sol en culture de maïs irrigué: approche fonctionnelle par la méthode des sacs de litière. Canadian Journal of Soil Science, 2000, 80 (2), pp.237-249. 10.4141/s99-055 . hal-03218275

\section{HAL Id: hal-03218275 \\ https://hal.science/hal-03218275}

Submitted on 5 May 2021

HAL is a multi-disciplinary open access archive for the deposit and dissemination of scientific research documents, whether they are published or not. The documents may come from teaching and research institutions in France or abroad, or from public or private research centers.
L'archive ouverte pluridisciplinaire HAL, est destinée au dépôt et à la diffusion de documents scientifiques de niveau recherche, publiés ou non, émanant des établissements d'enseignement et de recherche français ou étrangers, des laboratoires publics ou privés. 


\title{
Impact de produits phytopharmaceutiques sur les microarthropodes du sol en culture de maïs irrigué: approche fonctionnelle par la méthode des sacs de litière
}

\author{
J. Cortet et N. Poinsot-Balaguer \\ e-mail: Jerome.Cortet@univ-montp3.fr
}

\begin{abstract}
Cortet, J. et Poinsot-Balaguer, N. 2000. Impact de produits phytopharmaceutiques sur les microarthropodes du sol en culture de maïs irrigué: approche fonctionnelle par la méthode des sacs de litière. Can. J. Soil Sci. 80: 237-249. Les effets de deux herbicides (atrazine et alachlore) et deux insecticides (fipronil et carbofuran) ont été évalués sur la mésofaune du sol et la décomposition de la matière organique, dans un champ de maïs en conditions réelles de culture, en utilisant la méthode des sacs de litière. La méthode des sacs de litière est discutée. Elle a été jugée pertinente pour les études in situ, où le problème le plus délicat concerne le choix de témoins permettant d'apprécier l'impact réel des produits phytopharmaceutiques, à condition de respecter certains impératifs d'utilisation. L'étude de différents groupes taxonomiques rassemblés en groupes fonctionnels permet d'interpréter la dynamique de colonisation des sacs par les microarthropodes, celle-ci pouvant être ou non modifiée par les produits phytopharmaceutiques utilisés. Dans cette étude, concernant les herbicides, seul l'alachlore semble exercer une action différenciée sur la colonisation des sacs par la mésofaune. De même, l'insecticide fipronil agit de façon significative sur la dynamique de colonisation des sacs par certains groupes appartenant à la mésofaune du sol.
\end{abstract}

Mots clés: Produits phytopharmaceutiques, maïs, microarthropodes du sol, groupes fonctionnels, relations trophiques, sacs de litière

Cortet, J. and Poinsot-Balaguer, N. 2000. Impact of phytopharmaceutical products on soil microarthropods in an irrigated maize field: the use of the litter bag method. Can. J. Soil Sci. 80: 237-249. The effects of two herbicides (atrazine and alachlore) and two insecticides (fipronil and carbofuran) were evaluated on soil mesofauna and organic matter decomposition, in a maize field with normal culture conditions, using the litter-bag method. The litter-bag method was discussed and considered to be adapted for this type of in situ study, especially under normal culture conditions, where it is difficult to find real control plots. However its adaptability is conditioned by some utilisation factors. The litter-bag colonization and modifications of this colonization by phytopharmaceuticals were interpreted using functional groups classification. Except for alachlore, herbicides appeared to have no differentiated effect on mesofauna. Accordingly, fipronil significantly affects the dynamics of bag colonization by selected groups belonging to soil mesofauna.

Key words: Phytopharmaceuticals, maize, soil microarthropods, functional groups, trophic relationships, litter-bags

L'évaluation en microcosmes ou mésocosmes des effets létaux et/ou sublétaux des produits phytopharmaceutiques sur les microarthropodes du sol est généralement basée sur la réaction d'une seule espèce, et donne une image trop simplifiée du système, ignorant souvent complètement les relations interspécifiques (Fabián et Petersen 1994; Krogh 1995; Krogh et Pedersen 1995; Martikainen 1996; Wiles et Frampton 1996; Sarkar et al. 1997; Stark et al. 1997).

Même si ce type d'expérimentation commence à aborder le rôle des relations trophiques, et notamment les relations proies-prédateurs dans le transfert des pesticides le long des chaînes alimentaires, il ne permet pas la compréhension de certains phénomènes se déroulant à des niveaux supérieurs de complexité et possède peu de signification écologique (Petersen et Gjelstrup 1995; Hamers et Krogh 1997). Les études in situ peuvent donc seules répondre à ces préoccupations, malgré la lourdeur des protocoles et les contraintes liées à la mise en culture (Sabatini et al. 1979; Mallow et al. 1985).
Le but des recherches exposées est de mettre au point des méthodes fiables et reproductibles d'évaluation de l'impact de produits phytopharmaceutiques sur des paramètres biologiques en condition réelles d'exploitation d'une culture de maïs, ce qui signifie la prise en compte d'un passé cultural diversifié. La présente étude propose l'utilisation de la méthode des sacs de litière, qui vient d'être évaluée comme pertinente par rapport à différents critères écotoxicologiques (Kula et Römbke 1998). En particulier, elle permet d'évaluer la décomposition de la matière organique, paramètre considéré comme essentiel pour comprendre les effets des pesticides sur le fonctionnement biologique des sols.

Cette méthode permet en outre de mesurer la dynamique de colonisation de sacs, remplis de litière et déposés sur le sol, par la faune du sol. Même si elle n'évite pas totalement les problèmes liés à la rémanence des sols, puisque les animaux qui colonisent ces sacs viennent du sol où des phénomènes de sélection ont pu s'exercer antérieurement à 


\begin{tabular}{|c|c|c|c|c|c|}
\hline \multicolumn{3}{|c|}{ bloc herbicide } & \multicolumn{3}{|c|}{ bloc insecticide } \\
\hline témoinH & atrazine & alachlore & fipronil & témoinI & carbofuran \\
\hline alachlore & témoin $\mathrm{H}$ & atrazine & témoinI & fipronil & carbofuran \\
\hline témoinH & atrazine & alachlore & carbofuran & témoinI & fipronil \\
\hline $\begin{array}{c}\text { AS } \\
\text { atrazine }\end{array}$ & $\begin{array}{c}\text { AS } \\
\text { alachlore }\end{array}$ & témoinH & $\begin{array}{l}\text { AS } \\
\text { fipronil }\end{array}$ & $\begin{array}{l}\text { AS } \\
\text { carbofuran }\end{array}$ & $\begin{array}{c}\mathrm{AS} \\
\text { témoinI }\end{array}$ \\
\hline
\end{tabular}

Fig. 1. plan du champ expérimental et dénomination des parcelles. AS: parcelles ayant servi aux analyses de résidus de pesticides dans le sol. notre étude (Sabatini et al. 1979; Fratello et al. 1985), le sac de litière est un milieu parfaitement standard puisque rempli d'une seule et même matière organique. Cette méthode mesure les "préférences" des microarthropodes pour le milieu qui leur semble le plus favorable, et propose ainsi comme hypothèse de départ une variabilité de ces "préférences" en fonction du traitement subi par la matière organique ensachée. Elle permet, de plus, d'appréhender les stratégies adoptées par les taxons rencontrés et d'étudier les relations trophiques des différents groupes fonctionnels impliqués, tout en évaluant les modifications engendrées par les pesticides sur ces relations.

Deux herbicides (atrazine et alachlore), ainsi que deux insecticides (fipronil et carbofuran) sont testés pour valider la méthode. Alachlore et fipronil ne semblent pas avoir fait l'objet d'étude analogue. L'atrazine principalement et le carbofuran ont par contre été étudiés mais la plupart des expérimentations n'ont pas eu lieu en conditions réelles de culture (Fox 1964; Popovici et al. 1977). Les résultats sont cependant intéressants si l'on se place dans une approche plus générale de l'impact de perturbations sur les relations entre groupes fonctionnels.

\section{MATÉRIEL ET MÉTHODES}

\section{Site d'étude}

Le site d'étude se localise sur la ferme expérimentale du lycée agricole de La Côte Saint-André (Département de l'Isère, Région Rhône-Alpes, France). Le climat est de type tempéré, modérément méditerranéen: durant la période d'étude, d'avril à octobre, les précipitations moyennes mensu- elles les plus faibles sont enregistrées en juillet (57 mm), et les plus élevées en septembre (94 mm); les températures mensuelles moyennes, durant la même période, sont minimales en avril $\left(9.3^{\circ} \mathrm{C}\right)$ et maximales en juillet $\left(19.3^{\circ} \mathrm{C}\right)$. Les expérimentations se déroulent sur un champ carré de $80 \mathrm{~m}$ de côté, cultivé en maïs (Zea mays L.) depuis 8 ans, excepté l'année 1995 pendant laquelle du tabac (Nicotiana tabacum) a été planté. Le sol est de type brun lessivé (Food and Agriculture Organisation: Orthic luvisol - United States of America: Typic Hapludalf) sur roche mère fluvioglaciaire, avec un horizon A1-Ap (16\% de sables grossiers, $15.9 \%$ de sables fins, $16,5 \%$ de limons grossiers, $29 \%$ de limons fins, $20.6 \%$ d'argiles, $20 \mathrm{~g} \mathrm{~kg}^{-1}$ de matières organiques, $\mathrm{C} / \mathrm{N}: 12$ ) de 5 à $20 \mathrm{~cm}$. de profondeur, et un horizon Bt (10.1\% de sables grossiers, $11.5 \%$ de sables fins, $20.2 \%$ de limons grossiers, $29.2 \%$ de limons fins, $27.9 \%$ d'argiles, $11 \mathrm{~g} \mathrm{~kg}^{-1}$ de matières organiques, C/N: 9) de 40 à $60 \mathrm{~cm}$ de profondeur. Les semis de maïs (variété Marista non traitée Gaucho) ont eu lieu le 2 mai 1997. Tous les travaux effectués ont été conformes à la bonne pratique agricole, avec notamment un amendement en $\mathrm{N}$ de $240 \mathrm{~kg} \mathrm{ha}^{-1}$, en $\mathrm{P}$ de $45 \mathrm{~kg} \mathrm{ha}^{-1}$ et en $\mathrm{K}$ de $300 \mathrm{~kg} \mathrm{ha}^{-1}$. Le 16 mai, le maïs était au stade 2 feuilles. Le stade 6 feuilles a été atteint le 6 juin. La récolte a eu lieu dans la semaine du 20 au 24 octobre 1997. Le maïs a été irrigué régulièrement pendant les mois estivaux.

\section{Protocole expérimental sur le terrain}

Un champ a été divisé en deux grands blocs, le premier pour l'essai "herbicide" et le second pour l'essai "insecticide" 


\begin{tabular}{|c|c|c|c|c|}
\hline \multirow[b]{2}{*}{ Dénomination des parcelles } & \multicolumn{2}{|c|}{ traitements des parcelles } & \multicolumn{2}{|c|}{ traitements des sacs de litière } \\
\hline & herbicides & insecticides & herbicides & insecticides \\
\hline atrazine & $\begin{array}{c}\text { atrazine }+ \\
\text { pyridate }\end{array}$ & aucun & $\begin{array}{c}\text { atrazine }+ \\
\text { pyridate }\end{array}$ & aucun \\
\hline alachlore & $\begin{array}{c}\text { alachlore }+ \\
\text { pyridate }\end{array}$ & aucun & $\begin{array}{c}\text { alachlore }+ \\
\text { pyridate }\end{array}$ & aucun \\
\hline témoin $\mathrm{H}$ & pyridate & aucun & pyridate & aucun \\
\hline carbofuran & $\begin{array}{c}\text { atrazine }+ \\
\text { dimethenamide }\end{array}$ & carbofuran & $\begin{array}{c}\text { atrazine }+ \\
\text { dimethenamide }\end{array}$ & aucun \\
\hline fipronil & $\begin{array}{c}\text { atrazine }+ \\
\text { dimethenamide }\end{array}$ & fipronil & $\begin{array}{c}\text { atrazine }+ \\
\text { dimethenamide }\end{array}$ & fipronil \\
\hline témoin I & $\begin{array}{c}\text { atrazine }+ \\
\text { dimethenamide }\end{array}$ & aucun & $\begin{array}{c}\text { atrazine }+ \\
\text { dimethenamide }\end{array}$ & aucun \\
\hline
\end{tabular}

(figure 1). Chaque bloc a été lui-même divisé en 12 parcelles élémentaires, dont chacune comportait 16 rangs de maiis et mesurait $12.8 \times 20 \mathrm{~m}$.

Sur le bloc "herbicide" ont été comparés l'atrazine (famille des triazines, chloro-2 éthylamino-4 isopropylamino-6 triazine-1,3,5) et l'alachlore (famille des amides, chloro-2N-(diéthyl-2,6 phényl) N-méthoxyméthyl acétamide). Chaque traitement a été testé sur quatre parcelles élémentaires (soit huit parcelles traitées). Aucun insecticide ni fongicide n'a été utilisé dans le bloc "herbicide", mais la surface trop grande des parcelles ne permettant pas la suppression des adventices de façon manuelle, une zone sans aucun herbicide n'a pu être envisagée. C'est pourquoi les quatre parcelles témoin du bloc "herbicide" (témoinH), tout comme l'ensemble des 12 parcelles du bloc "herbicide", ont été traitées avec un autre herbicide, le pyridate (famille des diazines, 0-(6-chloro-3 phényl-4-pyridazinyl) carbothiolate d'n-octyl). L'atrazine et l'alachlore, ont été aspergés après les semis et avant l'apparition des feuilles (postsemisprélevée), à raison de $1 \mathrm{~kg} \mathrm{ha}^{-1}$ pour l'atrazine et $2.4 \mathrm{~kg} \mathrm{ha}^{-1}$ pour l'alachlore. Le pyridate a été aspergé au stade 6 feuilles (postlevée), à raison de $900 \mathrm{~g} \mathrm{ha}^{-1}$. Ces quantités correspondent aux doses recommandées pour ce type d'activité agricole [Association de Coordination Technique Agricole (ACTA) 1998].

Dans le bloc "insecticide" ont été testés le carbofuran (famille des carbamates, N-méthylcarbamate de diméthyl2,2 dihydro-2,3 benzofurannyle-7) et le fipronil (famille des phénylpyrazoles, 5-amino-1-(2,6 dichloro-x, $\mathrm{x}, \mathrm{x}$,-trifluro-ptolyl)-4-trifluorométhylsulfinyl-pyrazole-3-carbonitrile). Chaque traitement a été réalisé sur quatre parcelles élémentaires (soit huit parcelles traitées). Le carbofuran a été appliqué dans la raie de semis à l'aide du microgranulateur du semoir, avant semis, à raison de $600 \mathrm{~g} \mathrm{ha}^{-1}$. Le fipronil a été appliqué au sol avant le semis à l'aide du pulvérisateur, à raison de $200 \mathrm{~g} \mathrm{ha}^{-1}$, et a ensuite été incorporé mécaniquement au sol. Il faut noter que ce travail du sol a été seulement réalisé sur l'ensemble du bloc "insecticide". Les quantités d'insecticides employées correspondent aux doses recommandées pour ce type d'activité agricole (ACTA 1998). Dans le bloc "insecticide", le témoin était constitué par les quatre parcelles restantes non traitées, mais sur lesquelles deux herbicides (atrazine et dimethenamide (famille des amides, chloro-N((1-méthyl-2-méthoxy)éthyl)$\mathrm{N}$-(2,4-diméthyl-thien-3 yl) acétamide)) ont été épandus, tout comme sur les 12 parcelles du bloc "insecticide", à raison respectivement de $1 \mathrm{~kg} \mathrm{ha}^{-1}$ et de $1.44 \mathrm{~kg} \mathrm{ha}^{-1}$, afin d'éliminer les adventices.

L'ensemble de ces interventions est résumé dans le tableau 1.

\section{Les résidus de pesticides dans le sol}

Les prélèvements de sol ont été effectués dans les parcelles jouxtant le chemin nord (notées AS sur la figure 1). Trois séries ont été faites: avant semis, 1 mo et 4 mo après les semis (ces analyses ont été menées par le service central d'analyses du Centre National de la Recherche Scientifique de Vernaison, france).

Les pesticides ont été extraits par $100 \mathrm{~mL}$ d'un mélange d'acétone et de méthanol $(50 / 50 \mathrm{w} / \mathrm{w})$ au Sohxlet $(2 \mathrm{~h}$ l'échantillons trempe dans la solution, 2 h l'échantillon baigne dans la vapeur). La solution résultante a été séchée sur $\mathrm{Na}_{2} \mathrm{SO}_{4}$ puis évaporée à l'évaporateur rotatif. Le résidu a été repris par $5 \mathrm{ml}$ de chloroforme et analysé par chromatographie en phase gazeuse couplée à la spectrométrie de masse (Hernandez et al. 1990).

\section{La faune}

La méthode utilisée est adaptée de celle des sacs de litière (litter-bag) (Crossley et Hoglund 1962). 4,5 g de feuilles de maïs séchées, non traitées au départ, ont été introduits dans des sacs en Nylon de $12 \times 12 \mathrm{~cm}$ et de maille $4 \mathrm{~mm}$. Trente sacs ont été déposés à plat à la surface du sol, au moment des semis, au centre des parcelles, au niveau des rangs 7 et 8 (15 sacs par rang, au milieu du rang, en ligne séparés de $30 \mathrm{~cm}$ les uns des autres). Le sol était nu au moment des semis, la plupart des résidus de maïs issus de la précédente récolte ayant été enfouis au moment du labour. Les sacs de litière ont reçu les mêmes doses de matière active que les parcelles sur lesquelles ils ont été déposés, excepté dans les parcelles traitées au carbofuran. En effet, cet insecticide étant introduit directement dans le sol et non sur le sol où se trouvent les sacs de litière, ceux-ci n'ont pas directement reçu la matière active. 
Cinq sacs de litière par parcelle ont été relevés les 20 mai, 18 juin, 2 juillet, 12 août, 11 septembre et 3 octobre 1997, mis dans des sacs en plastique hermétiques et acheminés au laboratoire. Les sacs relevés en mai, juillet, août et octobre ont servi pour l'extraction de la mésofaune par la méthode dite de Berlèse (1905), conservée dans l'alcool à $60^{\circ}$, et comptée sous la loupe binoculaire.

Les différents taxons de microarthropodes ont été classés dans deux groupes fonctionnels qui correspondent à deux niveaux trophiques: les microphytophages-détritiphages et les prédateurs. Cette division est toutefois arbitraire: de nombreux microarthropodes sont en effet omnivores et peuvent intervenir à différents niveaux (Andren et al. 1990). Notons qu'immatures et adultes ont été confondus.

Parmi les microphytophages et détritiphages ont été distingués:

- les cinq familles de collemboles (Entomobryidae, Isotomidae, Poduridae, Sminthuridae et Onychiuridae)

Parmi les prédateurs:

- la cohorte des Gamasina (sous-ordre des Mésostigmates)

- la cohorte des Uropodina (sous-ordre des Mésostigmates)

- le groupe des Aranéides (sous-classes des Araignées et Opilions)

Plusieurs taxons peuvent être considérés comme "mixtes", c'est à dire qu'ils comprennent à la fois des détritiphagesmicrophytophages et des prédateurs:

- le sous-ordre des Oribates. Toutefois, étant donné leur forte abondance, et bien qu'il soit connu que certaines espèces soient également nématophages (Travé et al. 1996), les Oribates peuvent être ici associés aux microphytophages-détritiphages.

- le sous ordre des Prostigmates

- le groupe des Insectes Ptérygotes, qui englobe les larves de Diptères, Psocoptères, Thysanoptères, Homoptères, Hyménoptères et larves de Coléoptères. Larves de Diptères, Thysanoptères et Psocoptères peuvent être classés, comme le suggère Bachelier (1978), dans le groupe des microphytophages-détritiphages. Larves de coléoptères sont généralement placées dans le groupe fonctionnel des prédateurs car $80 \%$ des larves de coléoptères vivant dans le sol sont des prédateurs (Bachelier 1978).

- le groupe des Myriapodes, qui englobe les classes de Diplopodes, Chilopodes et Pauropodes. Ils ont été déterminés et classés au sein des groupes fonctionnels selon les critères établis par Geoffroy (1992, 1993), les Chilopodes en tant que prédateurs, Diplopodes et Pauropodes comme microphytophages-détritiphages.

Les collemboles ont été déterminés selon la classification établie par Gisin (1960). Les Oribates, Prostigmates et Mesostigmates, ont été déterminés et classés selon les critères établis par Krantz (1978). Les autres groupes ont été déterminés selon l'ouvrage de Coineau (1974).

Les abondances sont exprimées en nombre d'individus par gramme de matière organique dans les sacs. La teneur en matière organique des résidus de feuilles issus des sacs a été évaluée sur l'ensemble des prélèvements, grâce à la spectrométrie de réflexion infrarouge (Ibrahima et al. 1995).

\section{Traitements statistiques}

Les différents traitements phytopharmaceutiques à l'intérieur de chaque bloc ont été comparés deux à deux, pour chacun des groupes taxonomiques ainsi que pour les pourcentages de masse restante déterminés.

Ces différentes comparaisons ont été effectuées pour chacune des dates échantillonnées, grâce à l'analyse de variance (ANOVA, logiciel Statview, Statview Albacus Concepts 1997).

Les abondances moyennes ont été calculées pour tous les individus appartenant à différents groupes: Entomobrydae, Isotomidae, Poduridae, Onychiuridae, Sminthuridae, Oribates, Uropodidae, Gamasina, Prostigmates, Chilopodes, Diplopodes, Pauropodes, Insectes Ptérygotes, Aranéides (araignées et Opilions), pour chacune des 24 parcelles élémentaires (atrazine, alachlore, pyridate, fipronil, carbofuran et témoinI), et pour chaque date d'échantillonnage. Ces abondances ont été découpées en dix classes $(0,0$ à $0.2,0.2$ à 0.5 , 0.5 à 1,1 à 5,5 à 10,10 à 50,50 à 100,100 à $200,>$ à 200 par parcelle), ce qui permet de prendre en compte les groupes comportant de faibles effectifs (Bonin et Tatoni 1990). La matrice ainsi créée, comportant 96 lignes (chaque ligne correspondant à la moyenne des échantillons pour une parcelle élémentaire et un mois) et 14 colonnes (chaque colonne correspondant à un groupe de microarthropodes) a été utilisée pour effectuer une Analyse Factorielle des Correspondances (AFC, logiciel BIOMECO, Praxeme RetD 1997).

\section{RÉSULTATS}

\section{Résidus de pesticides dans le sol}

Trois remarques doivent être ajoutées concernant les produits phytopharmaceutiques. Elles rendent compte de la très grande difficulté à travailler en conditions réelles de culture. Elles n'ont, dans ce cadre, malheureusement pas pu être contournées:

- l'atrazine et le carbofuran étaient déjà utilisés régulièrement, chaque année sur les deux bloc de cette parcelle.

- des effets synergiques ou antagonistes peuvent avoir fonctionné, puisque différents produits sont souvent utilisés simultanément: pyridate-atrazine, pyridate-alachlore, fipronil-atrazine-dimethenamide, carbofuran-atrazine-dimethenamide. Il ne faut donc pas perdre de vue que les réponses observées peuvent avoir été amplifiées ou diminuées par rapport à celles que l'on pourrait obtenir en utilisant un produit seul.

- le mode d'administration du carbofuran est différent, puisqu'il a été introduit directement dans le sol, contrairement aux autres produits qui ont été aspergés. Les variables "produit phytosanitaire" et "mode d'administration de ce produit" sont donc dépendantes.

Les résultats seront donc analysés dans ce contexte et leur interprétation intégrera ces différents points.

Dans la parcelle "témoinI", les analyses effectuées avant traitement montrent une absence d'alachlore et de fipronil (tableau 2), mais une présence d'atrazine et de carbofuran. Ces résultats confirment que ces deux derniers pesticides étaient déjà présents dans le sol, avant la mise en place de l'échantillonnage. 

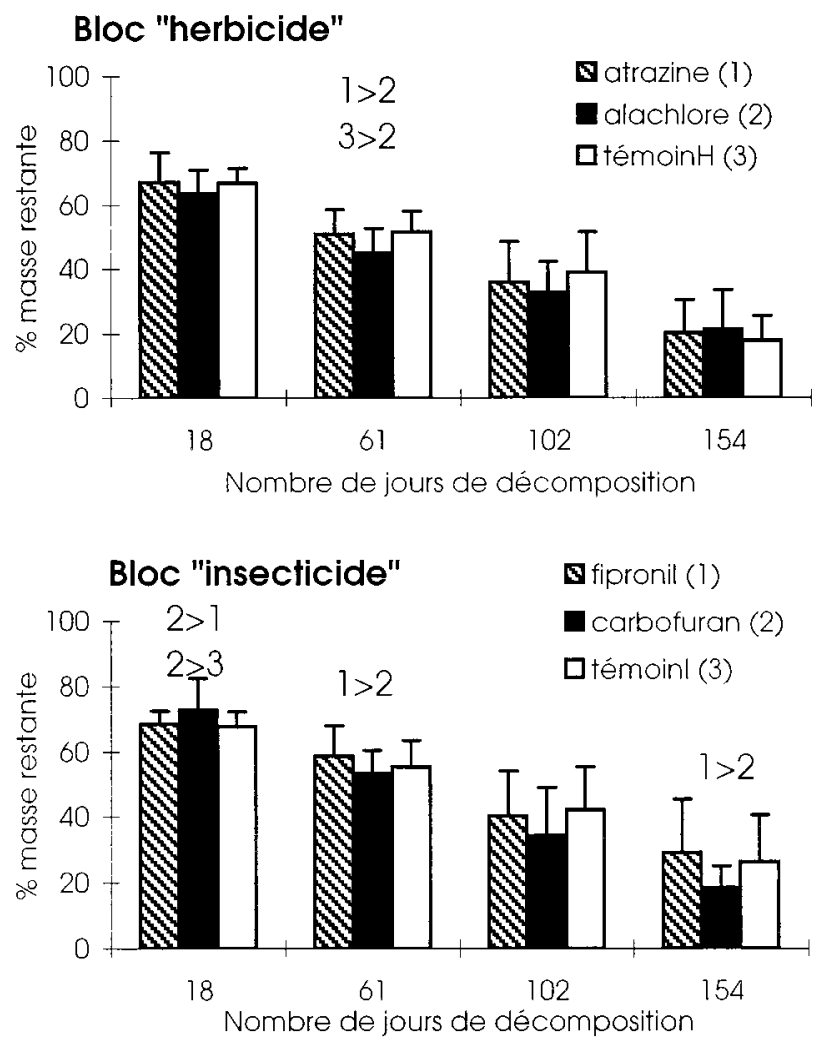

Fig. 2. Pourcentage de masse restante dans les sacs et différences entre traitements pour chacun des blocs (ANOVA sur 60 échantillons par mois par bloc: $P<0.05$ ).

Concernant l'alachlore, après traitement, les teneurs dans la parcelle "témoinI" sont très faibles, comparées à la parcelle "alachlore", ce qui laisse supposer que les parcelles non traitées à l'alachlore ont reçu très peu de cette substance.

En revanche, les teneurs en atrazine sont équivalentes dans la parcelle "témoinI" et la parcelle "atrazine". Ces résultats ne sont pas étonnants, puisque les parcelles "témoinI" ont également été traitées à l'atrazine, comme l'ensemble du bloc "insecticide". Ces résultats ne signifient donc pas que les parcelles traitées à l'alachlore ou les "témoinH" contiennent autant d'atrazine que les parcelles "témoinI". A priori, on peut plutôt supposer que les teneurs en atrazine dans les parcelles "alachlore" et "témoinH" sont équivalentes à celles rencontrées avant traitement dans les parcelles "atrazine" (soit environ $7 \mu \mathrm{g} \mathrm{kg}^{-1}$ ).

Concernant, le carbofuran, il est important de noter que la parcelle "témoinI" contient des quantités de matière active équivalentes à la parcelle "carbofuran". Après 1 mois, les quantités sont plus faibles dans la parcelle "témoinI", mais après 4 mois, on constate une absence de carbofuran dans la parcelle "carbofuran". En revanche, la parcelle "témoinI" contient encore du carbofuran après 4 mois, ce qui peut paraître assez surprenant, mais pourrait être expliqué par le mode d'administration du carbofuran sous forme de microgranulé, induisant une variabilité spatiale des teneurs en carbofuran dans le sol.
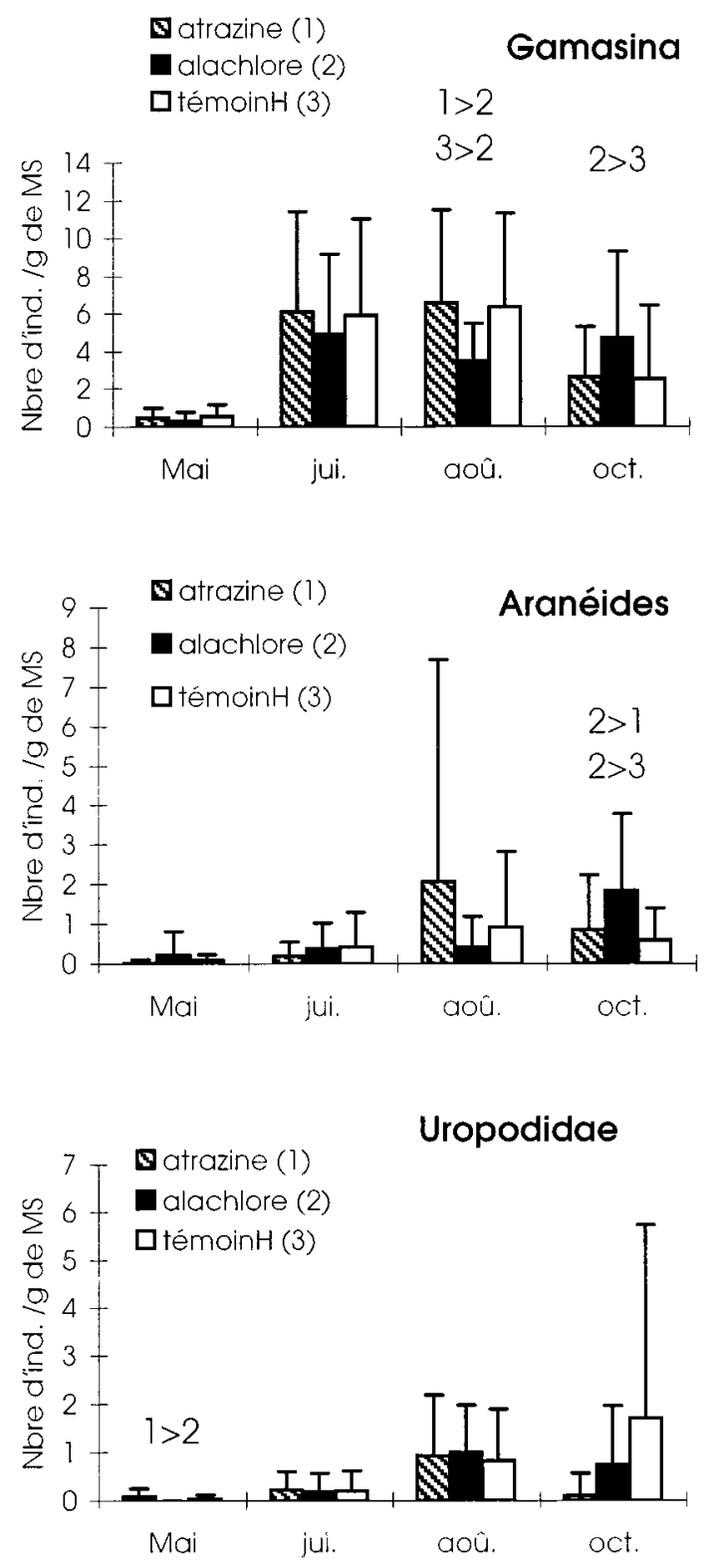

Fig. 3. Abondance des groupes zoologiques présentant des différences significatives entre les traitements dans le bloc "herbicide", selon la date échantillonnée (ANOVA sur 60 échantillons par mois; $P<0.05$.) MS: matière sèche.

Les quantités de fipronil sont nettement plus élevées dans la parcelle "fipronil" que dans la parcelle "témoinl", ce qui laisse supposer que les parcelles non traitées au fipronil ont reçu très peu de matières actives. Les résidus de fipronil observés dans les parcelles "témoinI" pourraient être dus à un apport extérieur par le vent, compte tenu de l'épandage par aspersion de cet insecticide.

\section{Pourcentages de masse restante dans les sacs}

La décomposition de la matière organique est rapide (figure 2). En effet, après 154 jours de décomposition, les pourcentages de masse restante varient de $17.8 \%$ ("témoinH") à $29.2 \%$ ("fipronil"). 

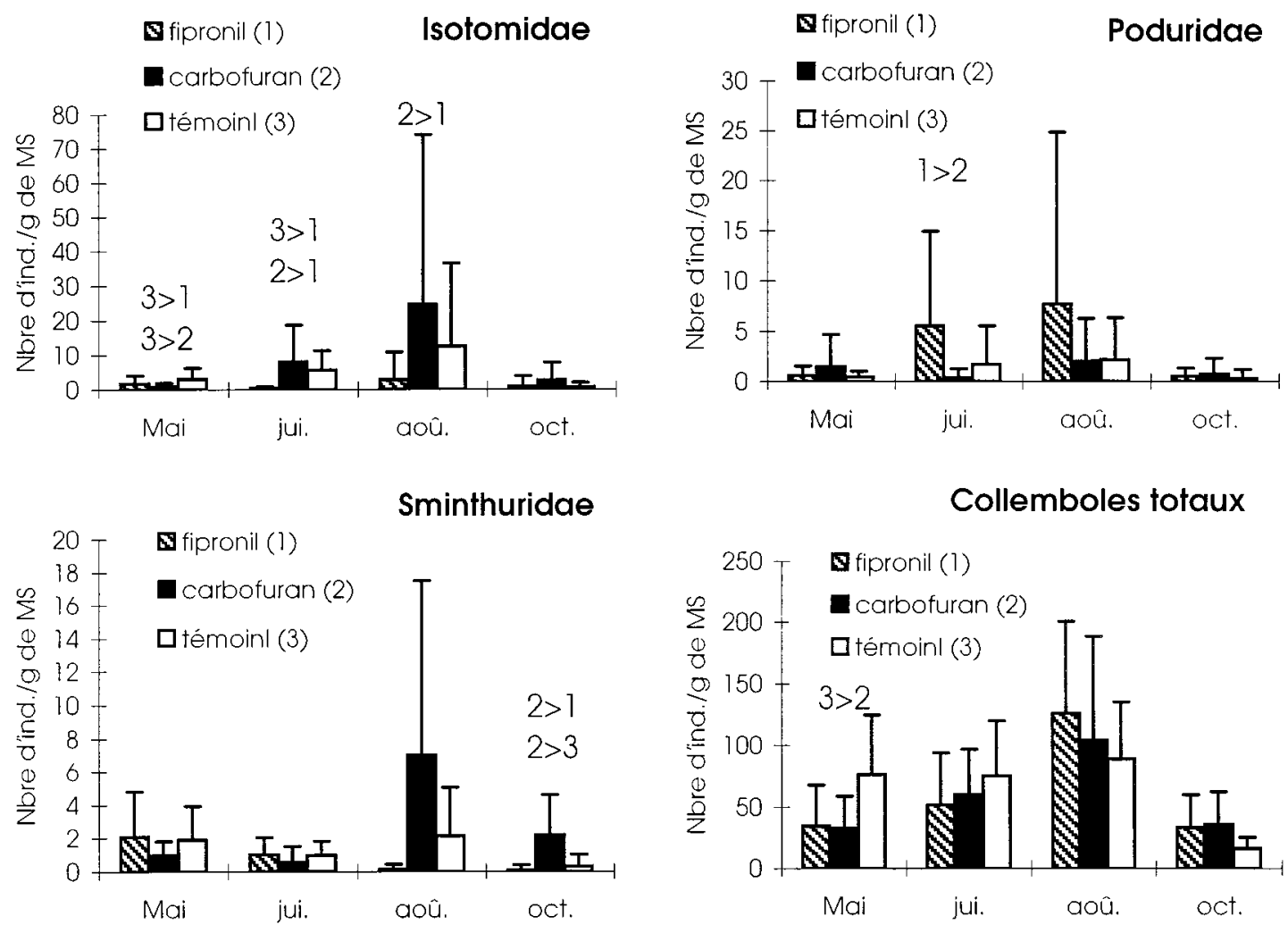

\section{Collemboles totaux}
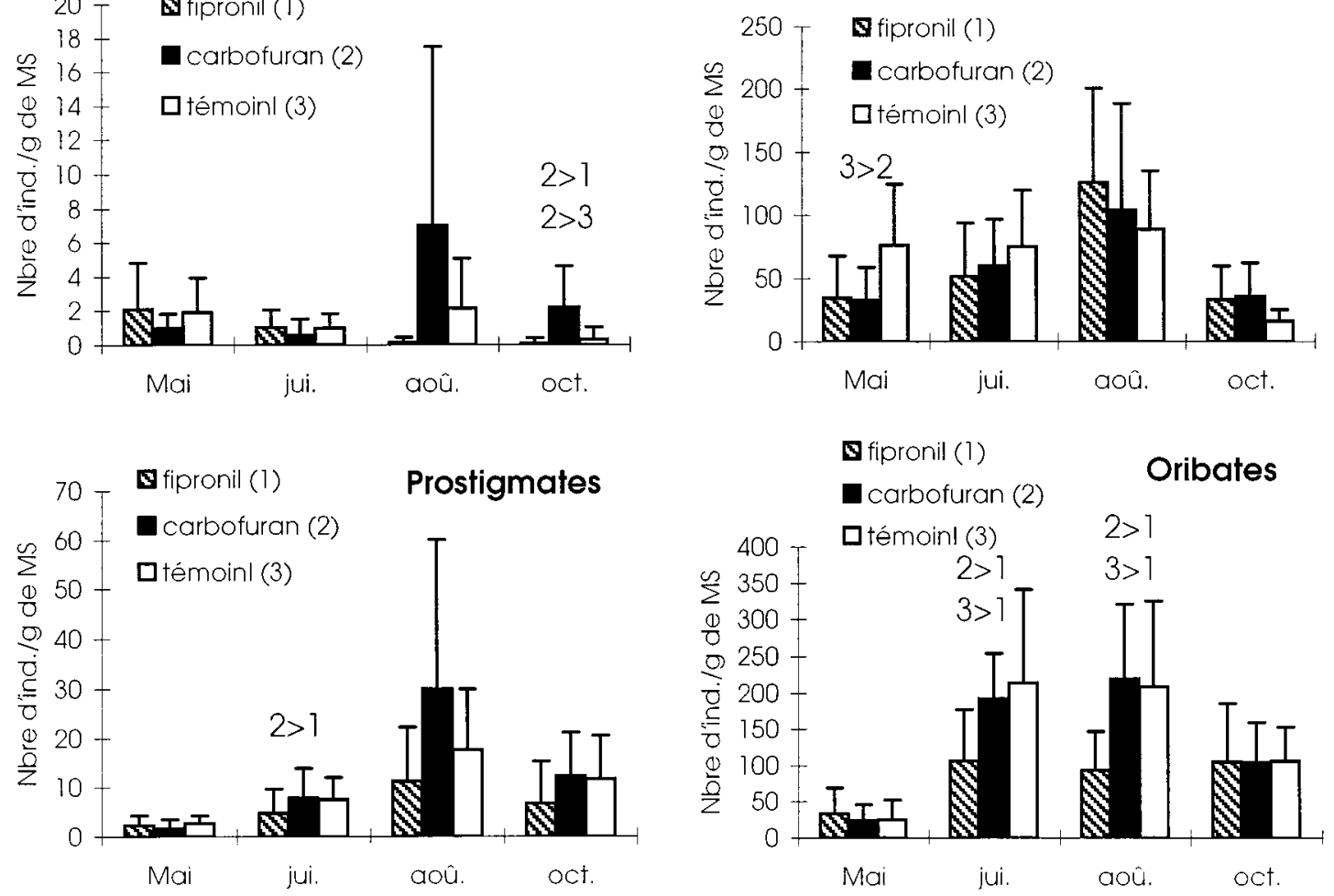

Fig. 4a. Abondance des groupes zoologiques présentant des différences significatives dans le bloc "insecticide", selon la date échantillonnée (ANOVA sur 60 échantillons par mois; $P<0.05$.) MS: matière sèche.

\section{Bloc "herbicide"}

Des différences significatives sont relevées entre les traitements au mois de juillet: en effet, après 61 jours de décomposition, les pourcentages de masses restantes sont plus faibles dans les parcelles "alachlore" que dans les parcelles du bloc "herbicides". Aucune différence n'est observée entre les parcelles "témoinH" et "atrazine". Au bout de 154 jours (octobre), aucune différence significative n'est observée entre les trois traitements.
Bloc "insecticide"

Des différences significatives sont relevées en mai, juillet et octobre. Elles varient en fonction de la date échantillonnée: au bout de 18 jours (mai), la décomposition est plus lente dans les parcelles "carbofuran" par rapport aux autres traitements; en revanche, après 61 et 154 jours, la décomposition est significativement plus rapide dans les parcelles "carbofuran" que dans celles traitées au fipronil. Aucune différence significative n'est relevée entre les parcelles "fipronil" et "témoinI". 

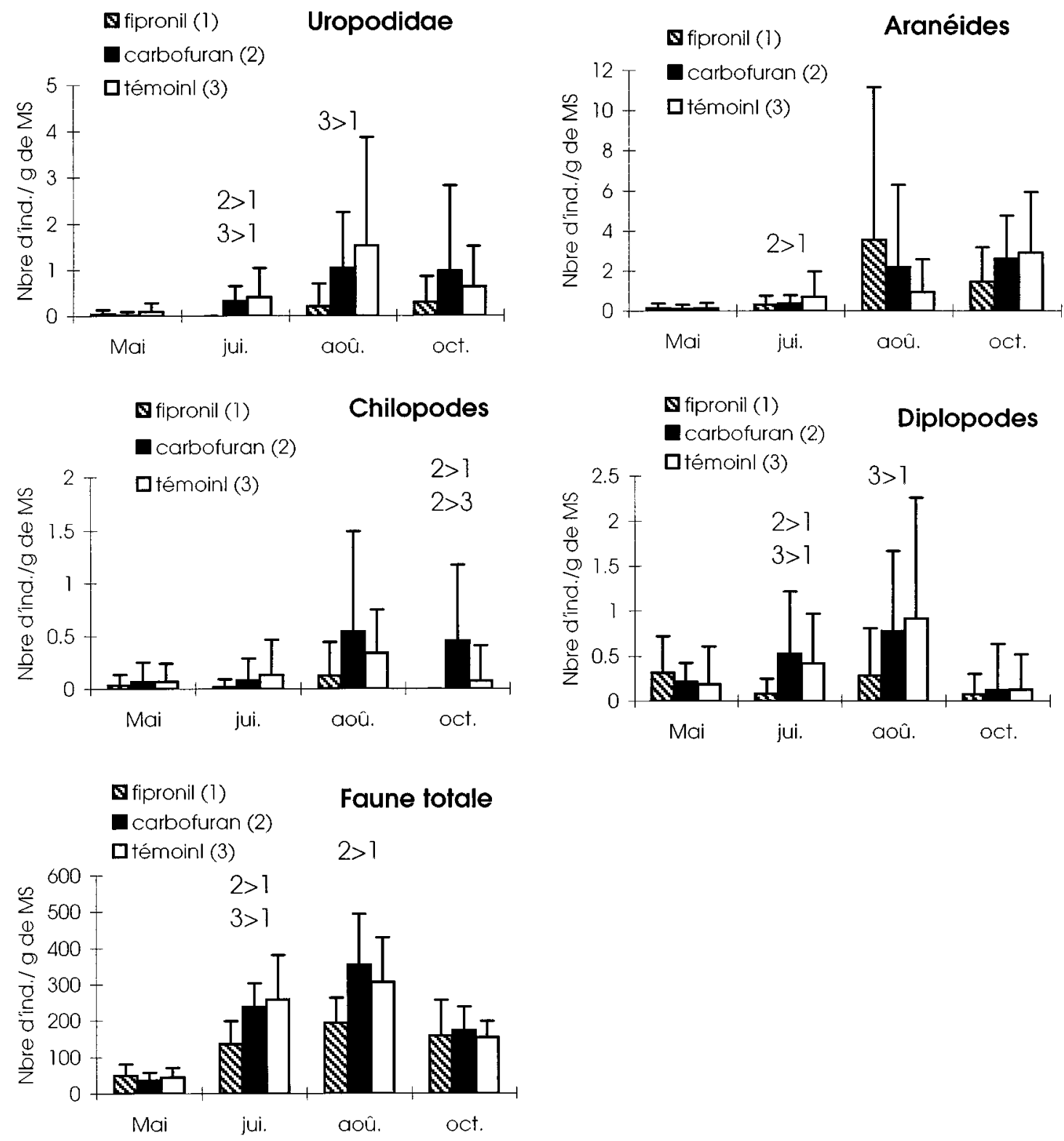

Fig. 4b. Abondance des groupes zoologiques présentant des différences significatives dans le bloc "insecticide", selon la date échantillonnée (ANOVA sur 60 échantillons par mois; $P<0.05$.) MS: matière sèche.

\section{Faune du sol}

Bloc "herbicide"

En mai, seuls les Uropodidae ont des abondances supérieures dans les parcelles traitées à l'atrazine que dans celles traitées à l'alachlore (figure 3). En juillet aucune différence significative n'est observée. En août, des abondances de Gamasina significativement plus faibles sont observées dans les parcelles traitées à l'alachlore que dans les autres parcelles. Le groupe trophique des prédateurs est moins abondant dans les parcelles traitées à l'alachlore que dans celles traitées à l'atrazine. En revanche, en octobre, les abondances sont plus élevées dans les parcelles traitées à l'alachlore que dans celles traitées à l'atrazine (Aranéides) ou "témoinH" (Gamasina, Aranéides, groupe trophique des prédateurs).

Aucun groupe de microphytophages ne montre de différence significative entre les traitements. Concernant les prédateurs, les différences sont plus marquées en août et octobre qu'aux autres moments de la saison. Il faut également remarquer qu'aucune différence significative de population des différents groupes étudiés n'est relevée entre les parcelles "atrazine" et "témoinH".

\section{Bloc "insecticide"}

En mai, les différences entre les traitements sont peu nombreuses. Seuls les Isotomidae sont significativement plus 


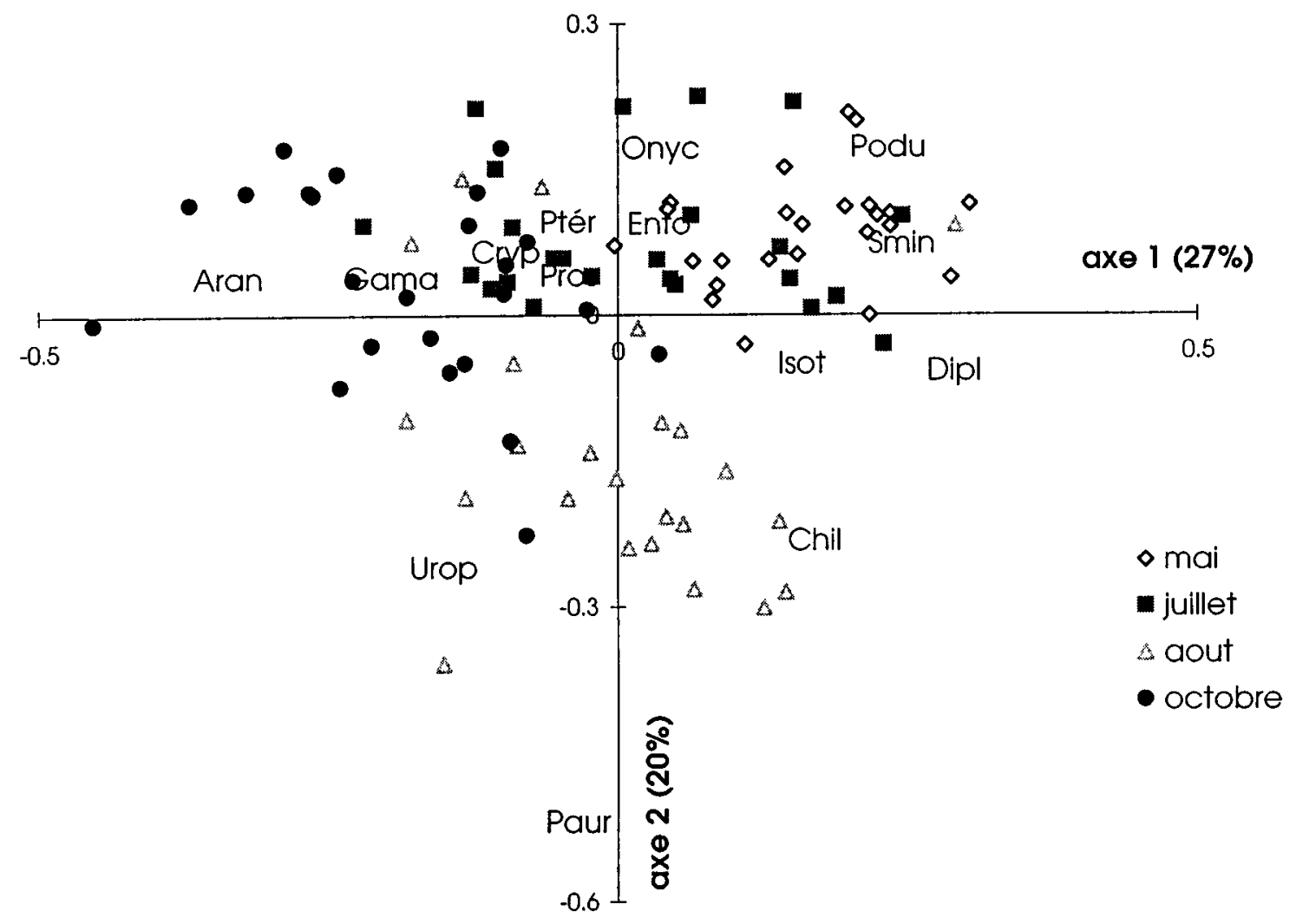

Fig. 5. Analyse factorielle des correspondances des 96 échantillons moyens et 14 groupes taxonomiques (plan formé par les axes 1 et 2 ). Ento: Entomobrydae. Isot: Isotomidae. Podu: Poduridae. Onyc: Onychiurudae. Smin: Sminthuridae. Gama: Gamasina. Urop: Uropodida. Cryp: Cryptostogmates. Pros: Prostignates. Chiol: Chilopoes. Dipl: Diplopodes. Paur: Pauropodes. Aran: Aranéides. Ptér: Insects Ptérygotes.

abondants dans les parcelles "témoinI" que dans celles traitées au carbofuran ou au fipronil (figures $4 \mathrm{a}$ et $4 \mathrm{~b}$ ). En juillet de nombreux groupes présentent des abondances plus élevées dans les parcelles "témoinI" que dans celles traitées au fipronil: Isotomidae, Oribates, Diplopodes, Uropodidae. Les deux groupes trophiques et le total des microarthropodes présentent également ces différences. En outre, les abondances sont plus élevées dans les parcelles traitées au carbofuran que dans celles traitées au fipronil pour les Isotomidae, Oribates, Diplopodes, Uropodidae, Prostigmates, Myriapodes et insectes ptérygotes. Seul les microphytophages de la famille des Poduridae sont plus abondants dans les parcelles traitées au fipronil que dans celles traitées au carbofuran. En août, certains groupes sont encore plus abondants dans les parcelles "témoinI" que dans celles traitées au fipronil; c'est le cas Oribates et des Diplopodes ainsi que des Uropodidae. De même, certains groupes présentent des abondances significativement plus élevées en "carbofuran" qu'en "fipronil" (Isotomidae, Oribates). Le total des microarthropodes présente aussi des abondances plus élevées dans les parcelles traitées au carbofuran que dans celles traitées au fipronil. En octobre, peu de différences sont remarquées. Toutefois, les abondances de certains taxons sont significativement plus élevées dans les parcelles traitées au carbofuran que dans celles traitées au fipronil (Sminthuridae, Prostigmates et Chilopodes) ou "témoinI" (Sminthuridae et Chilopodes).

Mai voit donc se distinguer les parcelles "témoinI", avec certains groupes plus abondants que dans les autres traitements. En revanche, juillet et août individualisent les parcelles traitées au fipronil, avec, excepté les Poduridae, des abondances plus faibles que dans les autres parcelles. Octobre individualise surtout les parcelles traitées au carbofuran avec des abondances plus élevées que les autres traitements.

\section{Analyse factorielle des correspondances}

Les trois premiers axes représentent $60 \%$ de l'information, ce qui est remarquable compte tenu du nombre de variables (14) et d'échantillons (96).

Le plan formé par les deux premiers axes décrit nettement un gradient temporel (figure 5). L'axe 1 (27\% de l'information) oppose clairement les prélèvements effectués en mai (côté positif de l'axe) à ceux effectués en octobre (côté négatif). Les groupes caractéristiques de mai sont essentiellement trois familles de collemboles (Sminthuridae, Poduridae et Isotomidae), mais aussi les Diplopodes. Tous ces groupes sont des microphytophages ou détritiphages. Les groupes caractéristiques d'octobre sont des prédateurs: Gamasidae non Uropodidae et Aranéides. L'axe 2 (20\% de l'information) 


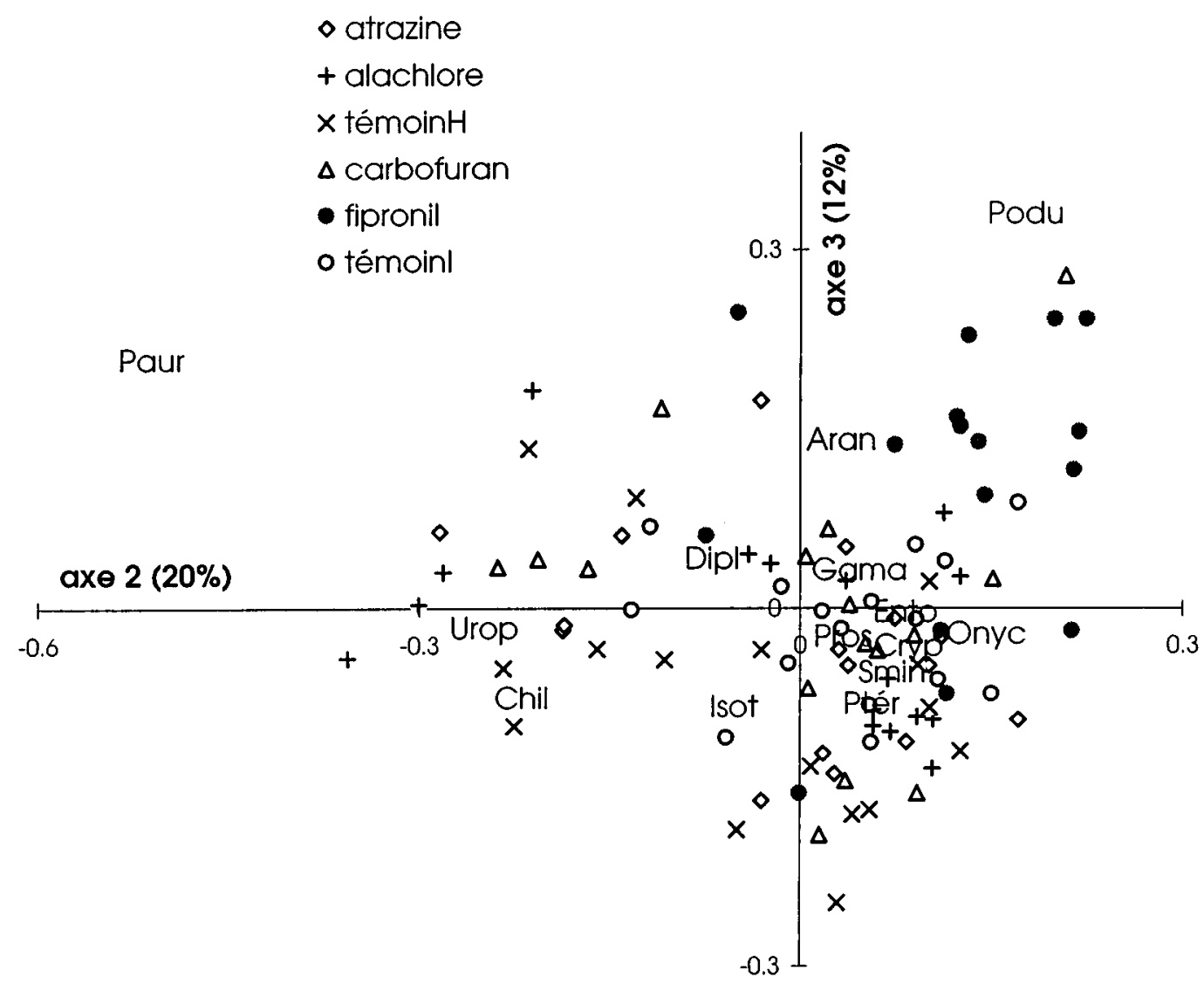

Fig. 6. Analyse factorielle des correspondances des 96 échantillons moyens et 14 groupes taxonomiques (plan formé par les axes 2 et 3 ). Ento: Entomobrydae. Isot: Isotomidae. Podu: Poduridae. Onyc: Onychiurudae. Smin: Sminthuridae. Gama: Gamasina. Urop: Uropodida. Cryp: Cryptostogmates. Pros: Prostignates. Chiol: Chilopoes. Dipl: Diplopodes. Paur: Pauropodes. Aran: Aranéides. Ptér: Insects Ptérygotes.

oppose les deux mois de prélèvements estivaux: juillet (côté positif de l'axe) et août (côté négatif). Le groupe des Poduridae domine en juillet, alors que les Uropodidae, les Chilopodes et Pauropodes caractérisent les prélèvements d'août. Une succession relativement nette de différents groupes est ainsi observée: groupes de microphytophages et détritiphages (collemboles et diplopodes) en mai et juillet, apparition de certains prédateurs comme les chilopodes et les Uropodidae en août puis apparition d'une seconde vague de prédateurs: les Aranéides et les Gamasina en octobre.

L'axe 3 (12\% de l'information) semble nettement discriminer les échantillons issus des parcelles traitées au fipronil. Ils sont caractérisés par une forte abondance de Poduridae. L'AFC confirme donc les résultats issus des analyses de variance, à savoir une nette individualisation des échantillons prélevés dans les parcelles traitées au fipronil (figure 6).

Les $40 \%$ d'information non expliqués par l'AFC peuvent être attribués à d'autres facteurs, comme la variation entre les parcelles d'un même traitement dues à des différences microstationnelles.
Certains groupes, comme les Poduridae, malgré leur faible abondance ont donc un poids important dans l'analyse factorielle des correspondances. C'est en effet une des caractéristique de l'AFC, qui permet, grâce au découpage en classes d'effectifs, aux espèces rares d'avoir un poids aussi important que les espèces abondantes. En outre, concernant le cas particulier des Poduridae, les résultats montrent que cette famille se comporte différemment des autres taxons, notamment par rapport au fipronil, ce qui tend à augmenter son poids sur l'AFC. Toutefois aucune de ces espèces peu abondantes n'est accidentelle, puisqu'elles sont présentes dans la plupart des relevés. A contrario, certains taxons numériquement importants, comme les Oribates, n'ont pas forcément un poids important sur les axes. Ce résultat signifie seulement que ces taxons ne sont pas ceux qui permettent de discriminer au mieux les échantillons, concernant les facteurs décrits par les axes 1, 2 et 3 . Il ne préjuge pas de l'importance de ces taxons à forts effectifs sur le fonctionnement de l'écosystème, par exemple sur le recyclage de la matière organique. 


\begin{tabular}{|c|c|c|c|c|c|}
\hline \multirow[b]{2}{*}{ parcelle } & \multirow[b]{2}{*}{ date } & \multicolumn{4}{|c|}{ pesticide analysé } \\
\hline & & alachlore & atrazine & carbofuran & fipronil \\
\hline \multirow[t]{3}{*}{ témoinI } & avant semis & absent & 7 & 6 & absent \\
\hline & 1 mois & absent & 12 & 3 & 8 \\
\hline & 4 mois & 4 & 27 & 8 & 6 \\
\hline \multirow[t]{2}{*}{ alachlore } & 1 mois & 84 & & & \\
\hline & 4 mois & 34 & & & \\
\hline \multirow[t]{2}{*}{ atrazine } & 1 mois & & 33 & & \\
\hline & 4 mois & & 33 & & \\
\hline \multirow[t]{2}{*}{ carbofuran } & 1 mois & & & 11 & \\
\hline & 4 mois & & & absent & \\
\hline \multirow[t]{2}{*}{ fipronil } & 1 mois & & & & 187 \\
\hline & 4 mois & & & & 120 \\
\hline
\end{tabular}

\section{DISCUSSION}

\section{Dynamique de colonisation des sacs de litière}

Il est connu que les premiers groupes qui apparaissent dans les sacs sont ceux qui présentent les caractères adaptés à une colonisation rapide des milieux. C'est le cas, dans notre étude, de certains groupes de collemboles comme les Poduridae ou les Sminthuridae. En effet, certaines espèces de collemboles ont un taux de reproduction élevé (grâce dans certains cas à leur capacité de parthénogénie) qui leur permet de se développer rapidement sur un nouveau substrat avant l'arrivée de groupes concurrents, comme par exemple les acariens Oribates dont les espèces ont généralement un taux de reproduction et un développement plus lent, et qui passent plutôt pour être des stratèges k (Crossley et al. 1992; Travé et al. 1996). Il convient toutefois de noter que certaines espèces d'Oribates peuvent se reproduire rapidement (Norton 1994). Ces derniers, même s'ils sont toujours abondants, sont en revanche proportionnellement moins nombreux en mai, au début de l'étude. En outre la qualité de la matière organique évolue dans le temps et participe ainsi à la sélection des différentes espèces en fonction de leurs préférences alimentaires. Ainsi, les Oribates et les Collemboles se nourrissent préférentiellement sur des fractions différentes de matière organique, les collemboles semblant préférer la matière organique fraîche, ce qui est également en accord avec nos résultats (Vreeken-Buijs et al. 1998). De même la qualité initiale de la matière organique exerce une sélection sur les espèces de microarthropodes rencontrées et notamment sur les collemboles (Cortet et Poinsot-Balaguer 1998a; Hågvar 1982). Logiquement, on observe, dans le cas étudié ici, que la colonisation des sacs par les prédateurs ne se fait, qu'après celle des microphytophages et des détritiphages dont on peut supposer qu'ils se nourrissent. Les variations saisonnières qui apparaissent pourraient être expliquées aussi par des facteurs climatiques; toutefois, le maïs ayant été irrigué régulièrement en été et les variations de températures étant faibles à cette époque, cette hypothèse est moins probable. Enfin la dynamique propre des différents taxons de microarthropodes est à prendre en compte. Certains groupes pouvant apparaître préférentiellement à certaines époques de l'année pour des raisons chronobiologiques.

\section{Effets des herbicides}

L'association atrazine-pyridate a une action comparable à celle du pyridate, employé seul des parcelles "témoinH", sur la colonisation des sacs par les microarthropodes du sol et leurs relations trophiques. Ces résultats rejoignent ceux de Wardle et al. (1995) sur les effets de la gestion des adventices par différentes techniques, en cultures de maïs: les cinq traitements utilisés (paillage, culture entre les rangs, sarclage manuel, atrazine $1.5 \mathrm{~kg} \mathrm{ha}^{-1}$, rimsulfuron $40 \mathrm{~g} \mathrm{ha}^{-1}+$ primisulfuron $40 \mathrm{~g} \mathrm{ha}^{-1}$ ) ne permettaient pas de dégager de réelles modifications au niveau des acariens et des collemboles, excepté une légère augmentation des effectifs dans les parcelles soumises à des traitements permettant un meilleur développement des adventices comme le paillage.

Cependant, dans une étude antérieure, nous avions évalué l'impact de l'atrazine sur une culture de maïs toujours à La Côte Saint André. Le protocole d'étude était identique à celui détaillé plus haut, mais avec des différences portant sur la taille des parcelles $\left(2 \mathrm{~m}^{2}\right)$ et l'utilisation d'un désherbage manuel dans la station témoin, qui elle-même ne supportait aucun traitement. Une augmentation significative et sélective de certaines espèces de Collemboles avait été observée dans les zones traitées (Cortet et Poinsot-Balaguer 1998b).

La comparaison avec d'autres expérimentations concernant l'atrazine est difficile. En effet, elles n'ont pas été réalisées dans les conditions réelles d'utilisation de cet herbicide en agriculture: les doses beaucoup plus élevées que celles préconisées et les protocoles expérimentaux introduisant des biais trop importants ne permettaient pas une évaluation réaliste des effets de cet herbicide. Ainsi Fox (1964), qui a utilisé des concentrations en atrazine largement supérieures aux nôtres $\left(8.8 \mathrm{~kg} \mathrm{ha}^{-1}\right.$ contre $\left.1 \mathrm{~kg} \mathrm{ha}^{-1}\right)$ dans une prairie, note une réduction de la densité de collemboles dans la station traitée par rapport à la station témoin dans laquelle il n'a pas effectué de désherbage manuel. Ses résultats intègrent donc un effet bénéfique du au développement de la flore adventice dans la parcelle témoin. De même, Popovici et al. (1977), qui a travaillé en cultures de maïs, avec des doses de 5 à $8 \mathrm{~kg}$ d'atrazine ha ${ }^{-1}$, et n'a effectué aucune intervention pour éliminer les adventices sur la parcelle témoin, a constaté des réductions très importantes des populations de collemboles dans la station traitée (plus de $70 \%$ inférieures en moyenne au bout d'un mois par rapport à la station non traitée).

Il convient toutefois de noter que les analyses de sol montrent clairement que l'atrazine était déjà présent sur notre site en début d'expérimentation. Des phénomènes de sélection peuvent donc avoir agi antérieurement à notre expérimentation.

L'accélération de la décomposition, observée en juillet en "alachlore", pourrait indiquer une activité biologique plus intense que dans les autres traitements. L'absence de différences significatives sur les principaux groupes de microphytophages laisse supposer qu'il s'agit plutôt d'une activité microbiologique. En effet, même si les doses utilisées ici sont faibles et peut-être même négligeables par rapport au pool de carbone labile du sol, il est connu que certains micro-organismes sont capables d'utiliser certains herbicides comme source d'énergie pour se développer (Neher 
1995). De même, certains prédateurs montrent des abondances plus faibles en mai et surtout en août en "alachlore" par rapport aux autres parcelles du bloc "herbicide". On peut supposer ici aussi, en l'absence d'effets sur les groupes de microphytophages, un effet direct létal sur ces prédateurs qui s'estompe ensuite. Mais ces prédateurs peuvent se nourrir sur d'autres composantes de la faune du sol, comme les nématodes, qui n'ont pas été abordés ici (Crossley et al. 1992; Zwart et Brussaard 1988), et qui peuvent avoir une grande importance dans les réseaux trophiques (Neher et Campbell 1994; Neher et al. 1995). Selon Gregorich et al. (1997) les sols labourés favoriseraient d'ailleurs les consommateurs de bactéries comme les nématodes.

La plus grande abondance de prédateurs rencontrée en octobre dans les stations "alachlore" par rapport aux autres traitements, pourrait s'expliquer au contraire par une stimulation de la reproduction. Ce phénomène, appelé hormesis, a déjà été observée en laboratoire à propos des effets de l'insecticide diméthoate sur le Gamaside Hypoaspis aculeifer (Krogh 1994). En effet, en condition de stress cet acarien aurait tendance à augmenter sa reproduction. Les mêmes observations ont été effectuées à propos des effets de l'insecticide cypermethrin sur l'araignée Pardosa armentata, espèce communément rencontrée dans les milieux agricoles d'Europe du nord (Toft et Jensen 1998). Il faut noter que les réponses "positives" au niveau populationnel, engendrées par une pollution sont d'ailleurs relativement courantes, notamment à faibles concentrations; elles peuvent être dues à des perturbations plus importantes à des niveaux inférieurs (Ramade 1992).

\section{Effets des insecticides}

L'application du fipronil amène des modifications importantes de la structure des communautés colonisant les sacs. Les groupes non affectés négativement sont ceux qui possèdent une ou plusieurs caractéristiques leur permettant de mieux résister à ce type de stress: ainsi les Aranéides et les Gamasina apparaissent tardivement en saison et se déplacent rapidement, et possèdent donc des capacités rapides de recolonisation à partir de zones non polluées (Koehler 1997; Greig-Smith 1988). En outre, ils se nourrissent surtout de collemboles (Laakso et al. 1995; Kaneko 1995; Koehler 1997; Berg et al. 1998), et ces derniers ne sont au total pas affectés significativement. La diversité des réponses offertes par les différentes familles de collemboles rencontrées dans nos échantillons a déjà été observée précédemment (Frampton 1997) et illustre parfaitement leurs stratégies adaptatives très diversifiées, tant au niveau de leur reproduction et développement, de leur taille et milieu de vie, que de leur régime alimentaire. Il est clair que les espèces à stratégie $\mathrm{r}$ (reproduction rapide parfois due à la parthénogénèse, faibles exigences de milieux, tendance à l'omnivorie, déplacements rapides) seront nettement avantagées, car elles pourront profiter de la baisse de compétitivité des espèces plus sensibles (Hågvar 1994; Petersen 1995). Les Oribates, composant la très grande majorité des microarthropodes rencontrés, semblent particulièrement sensibles et, sont vraisemblablement défavorisés par leur faible taux de reproduction et de développement, qui ne leur permet pas de recouvrer rapidement leur niveau initial.
Concernant les parcelles "carbofuran", une décomposition plus lente, avec certains groupes comme les Sminthuridae moins abondants que dans les parcelles "témoinI", est observée uniquement en mai. Le carbofuran ne semble donc pas affecter très durablement la colonisation des sacs par les microarthropodes. Il est vrai que les carbamates sont réputés moins toxiques pour la mésofaune du sol que la plupart des autres insecticides et notamment les organochlorés. Ainsi Krogh (1991), suite aux travaux de Broadbent et Tomlin (1982), signale que si le carbofuran est très toxique pour les vers de terre, les collemboles et acariens sont souvent stimulés à doses normales. Toutefois, il faut remarquer que le sol contenait déjà du carbofuran avant épandage et, excepté en mai, les différences de concentrations en matière active entre la parcelle "témoinI" et la parcelle "carbofuran" apparaissent peu différentes.

L'augmentation de la décomposition et les abondances plus importantes de certains groupes (pas toujours significatifs mais au moins tendanciels) en "carbofuran" par rapport aux parcelles "témoinI", à partir de juillet, pourraient être expliqués par le mode d'administration du carbofuran: rappelons que le produit a été introduit directement dans le sol et les feuilles de maïs contenues dans les sacs de litière n'ont pas été directement contaminées. Dans ce cas, les sacs sont identiques à ceux rencontrés dans les parcelles "témoinI". Selon Siedentop (1995), les sacs de litière non directement contaminés pourraient même agir comme des zones refuge. Les analyses de résidus ont par ailleurs montré qu'au bout de quatre mois la parcelle "témoinI" ayant servi aux analyses de sol, contenait encore du carbofuran, contrairement à la parcelle "carbofuran", ce qui pourrait également expliquer ces résultats.

\section{Intérêt et limites de la méthode}

La seule méthode qui donne une photographie exacte des microarthropodes dans le sol est la méthode des carottages de sol. Pour être exploitables, ces carottages doivent être nombreux, de préférence effectués dans des milieux homogènes, à différents niveaux de profondeur, et les résultats corrélés aux facteurs de l'environnement comme les paramètres pédoclimatiques. C'est donc une méthode très lourde à mettre en œuvre, qui ne permet pas une réelle approche fonctionnelle efficace concernant l'impact des produits phytopharmaceutiques.

La méthode des sacs de litière permet d'associer faune et activité biologique. Cette activité biologique peut d'ailleurs être contrôlée en jouant sur la taille de la maille qui permet ou non le passage de différents groupes faunistiques. Dans notre étude la maille est de $4 \mathrm{~mm}$ et permet un accès à la mésofaune, mais aussi à la macrofaune comme les vers de terre. Ces derniers sont d'ailleurs reconnus pour exercer une action significative sur la décomposition de la matière organique en sols cultivés: ainsi en champs de maïs, Bohlen et al. (1997) ont montré l'action significative du vers Lumbricus terrestris. Ces remarques pourraient expliquer les résultats concernant le fipronil qui induit un impact important sur les microarthropodes du sol, mais ne semble pas affecter la décomposition de la matière organique.

En outre, pour étudier les effets des produits phytopharmaceutiques en conditions réelles de culture, il nous semble 
préférable de placer les sacs en surface plutôt que de les enfouir, afin que la matière organique située dans ces sacs reçoive les même traitements aux mêmes doses que la surface du sol, tout au long de la saison de culture. C'est pourquoi, il est également préférable d'utiliser cette méthode pour tester des produits épandus par aspersion. Nous voyons d'ailleurs que nos résultats concernant le carbofuran sont ambigus: si la matière organique contenue dans le sac n'est pas traitée, elle risque de constituer un milieu particulièrement favorable et appétent comparativement à la matière organique du sol. Cette remarque rejoint celle de Kula et Römbke (1998), qui estiment que les sacs doivent être directement contaminés pour que la méthode soit efficace et réaliste.

Enfin, il faut noter que la méthode peut-être aussi utilisée pour évaluer d'autres types de stress (par exemple les épandages d'effluents ou de boues de stations d'épuration) et reste relativement flexible. Elle présente en outre l'avantage d'être facilement standardisable, facile à mettre en œuvre et peu coûteuse.

\section{CONCLUSION}

L'utilisation de la méthode des sacs de litière paraît prometteuse pour l'étude des produits phytopharmaceutiques en plein champ, au moins en ce qui concerne ceux épandus par aspersion et à condition de bien définir la notion de témoin.

Les réponses obtenues au niveau de la mésofaune font entrevoir les schémas complexes intervenants dans les flux de nutriments et les relations trophiques dans le sol. Les groupes zoologiques réagissent en fonction de leur sensibilité, elle-même conditionnée par un ensemble de caractères propre à chacun de ces groupes. Les microphytophages, notamment les collemboles et les Oribates semblent être des bioindicateurs plus pertinents que les prédateurs, car ils sont plus nombreux et moins mobiles. De plus, la variété des stratégies adaptatives dont ils sont capables permet d'obtenir des informations objectives sur l'impact de différents types de pollutions, élevés ou faibles, chroniques ou aigus. En outre, ils se situent au centre des réseaux alimentaires et sont donc susceptibles de réagir aux perturbations affectant aussi bien les micro-organismes dont ils se nourrissent, que celles affectant les prédateurs, dont ils constituent la principale ressource.

\section{REMERCIEMENTS}

Ce travail a été réalisé dans le cadre du programme PNETOX (Ministère de l'Environnement et de l'Aménagement du Territoire). Remerciements à Bernard Garino (Lycée Agricole de La Côte Saint André), René Bally (Ecologie microbienne du sol, CNRS Lyon) André Chabert (Association de Coordination Technique Agricole, Lyon), Richard Joffre et Jean-Marc Ourcival (Centre d'Ecologie Fonctionnelle et Evolutive, Montpellier) et Maurice Roux (Institut Méditerranéen d'Ecologie et Paléoécologie, CNRS Marseille) pour leur précieuse aide. Merci aussi à Sylvie Dupouyet (Institut Méditerranéen d'Ecologie et Paléoécologie, CNRS Marseille), ainsi qu'au GEOTOP (UQAM, Montreal) pour leur indispensable assistance technique. Remerciements enfin à Marie Claude Talin et Thierry Dutoit pour leur analyse critique sur ce travail.
Andren, O., Lindberg, T., Boström, U., Clarholm, M., Hansson, A. C., Johansson, G., Lagerlöf, J., Paustian, K., Persson, J., Petterson, R., Schnürer, J., Sohlenius, B. and Wivstad, M. 1990. Organic carbon and nitrogen flows. Dans O. Andren, T. Lindberg, K. Paustian, and T. Rosswall, eds. Ecology of arable land-organisms, carbon and nitrogen cycling. Ecol. Bull. 40: $85-126$.

Association de Coordination Technique Agricole. 1998. Index Phytosanitaire. ACTA, Paris, France.

Bachelier, G. 1978. La faune du sol: son écologie et son action. Initiations - Documentation techniques. 38, ORSTOM, Paris, France.

Berg, M. P., Kniese, J. P., Bedaux, J. J. M. and Verhoef, H. A. 1998. Dynamics and stratification of functional groups of microand mesoarthropods in the organic layer of a scots pine forest. Biol. Fertil. Soils 26: 268-284.

Berlèse, A. 1905. Apparacchio per raccogliere presto ed in gran numero piccoli arthropodi. Redia 2: 85-89.

Bohlen, P. J., Parmelee, R. W., McCartney, D. A. and Edwards, C. A. 1997. Earthworm effects on carbon and nitrogen dynamics of surface litter in corn agroecosystems. Ecol. Appl. 7: $1341-1349$.

Bonin, G. and Tatoni, T. 1990. Réflexions sur l'apport de l'analyse factorielle des Correspondances dans l'étude des communautés végétales et de leur environnement. Ecol. Mediterr. 16: 403-414.

Broadbent, A. B. and Tomlin, A. D. 1982. Comparison of two methods for assessing the effects of carbofuran on soil animal decomposers in cornfields. Environ. Entomol. 11: 1036-1042.

Coineau, Y. 1974. Introduction à l'étude des microarthropodes du sol et de ses annexes. Doin, Paris, France.

Cortet, J. and Poinsot-Balaguer, N. 1998a. Collembola populations under sclerophyllous coppices in Provence (France): comparison between two types of vegetation, Quercus ilex L. and Quercus coccifera L. Acta Oecol. 19: 413-424.

Cortet, J. and Poinsot-Balaguer, N. 1998b. Effects of atrazine on maize litter decomposition and litter-bag microarthropods colonization in an experimental maize field. Pages 1043-1044 dans Proceedings of the Sixth International FZK/TNO Conference on Contaminated Soil. Thomas Telford, London, RU.

Crossley, D. A. and Hoglund, M. P. 1962. A litter-bag method for the study of microarthropods inhabiting leaf litter. Ecology 43: 571-573.

Crossley, D. A., Mueller, B. R. and Perdue, J. C. 1992. Biodiversity of microarthropods in agricultural soils: relations to processes. Agric. Ecosyst. Environ. 40: 37-46.

Fabián, M. and Petersen, H. 1994. Short-term effects of the insecticide dimethoate on activity and spatial distribution of a soil inhabiting collembollan Folsomia fimetaria Linné (Collembola: Isotomidae). Pedobiologia 38: 289-302.

Fox, C. J. S. 1964. The effects of five herbicides on the numbers of certain invertebrate animals in grassland soil. Can. J. Plant Sci. 44: 405-409.

Frampton, G. K. 1997. The potential of collembola as indicators of pesticide usage: evidence and methods from the UK arable ecosystem. Pedobiologia 41: 179-184.

Fratello, B., Bertolani, R., Sabatini, M. A., Mola, L. and Rassu, M. A. 1985. Effects of atrazine on soil microarthropods in experimental maize fields. Pedobiologia 28: 161-168.

Geoffroy, J. J. 1992. Clés d'identification des classes de Myriapodes et des ordres de Chilopodes fréquents dans le sol et ses annexes (Myriapoda; Chilopoda). Millepattia 1: 23-37.

Geoffroy, J. J. 1993. Clés d'identification des ordres de diplopodes fréquents dans le sol (Myriapoda; Diplopoda). Millepattia 2: 4-28. 
Gisin, H. 1960. Collembolenfauna Europas. Museum d'Histoire Naturelle, Genève, Suisse.

Greig-Smith, P. W. 1988. The Boxworth experience: effects of pesticides on the fauna and flora of cereal fields. Pages 333-371 dans L. G. Firbank, N. Carter, J. F. Darbyshire, et G.R. Potts, eds. The ecology of temperate cereal fields. Blackwell Scientific Publications, Oxford, RU.

Gregorich, E. G., Carter, M. R., Doran, J. W., Pankhurst, C. E. and Dwyer, L. M. 1997. Biological attributes of soil quality. Pages 81-113 dans E. G. Gregorich and M. R. Carter, eds. Soil quality for crop production and ecosystem health. Elsevier Science Publishers, Amsterdam, Pays-Bas.

Hågvar, S. 1982. Collembola in Norwegian coniferous forest soils. II. Relations to soil chemistry. Pedobiologia 24: 255-296.

Hågvar, S. 1994. Log-normal distribution of dominance as an indicator of stressed soil microarthropod communities ? Acta Zool. Fenn. 195: 71-0.

Hamers, T. and Krogh, P. H. 1997. Predator-prey relationships in a two-species toxicity test system. Ecotoxicol. Environ. Saf. 37: 203-212.

Hernandez, F. H., Grases, J. M., Beltran, J. and Sancho, V. 1990. Comparative study of different residue method for the determination of pesticides in fruit sample by gaz chromatography. Chromatographia 29: 159-466.

Ibrahima, A., Joffre, R. and Gillon, D. 1995. Changes in litter during the initial leaching phase: an experiment on the leaf litter of Mediterranean species. Soil Biol. Biochem. 27: 931-939.

Kaneko, N. 1995. Composition of feeding types in oribatid mite communities in forest soils. Acta Zool. Fennica 196: 160-161.

Koehler, H. H. 1997. Mesostigmata (Gamasina, Uropodina), efficient predators in agroecosystems. Agric. Ecosyst. Environ 62: 105-117.

Krantz, G. W. 1978. A manual of acarology. Oregon State University Book Stores, Corvallis, OR.

Krogh, P. H. 1991. Pertubation of the soil microarthropod community with the pesticides benomyl and isofenphos. Pedobiologia 35: 71-88.

Krogh, P. H. 1994. Microarthropods as bio-indicators, a study of disturbed populations. PhD thesis, University of Aarhus, Ministry of the Environment and Energy, National Environment Institute, Danemark.

Krogh, P. H. 1995. Does a heterogeneous distribution of food or pesticide affect the outcome of toxicity tests with collembola? Ecotoxicol. Environ. Saf. 30: 158-163.

Krogh, P. H. and Pedersen, M. B. 1995. Laboratory toxicity testing with Collembola. Pages 39-58 dans H. Lokke, ed. Effects of pesticides on Meso- and Microfauna in soil. Danish Environmental Protection Agency, Copenhagen, Danemark.

Kula, C. and Römbke, J. 1998. Evaluation of soil Ecotoxicity tests with fonctional endpoints for the risk assesment of plant protection products. Environ. Sci. Pollut. Res. 5: 55-60.

Laakso, J., Salminen, J. and Setälä, H. 1995. Effects of abiotic conditions and microarthropod predation on the structure and function of soil animal communities. Acta Zool. Fennica 196: 162-167. Mallow, D., Snider, R. J. and Robertson, L. S. 1985. Effects of different management practices on collembola and acarina in corn production systems. Pedobiologia 28: 115-131.

Martikainen, E. 1996. Toxicity of dimethoate to some soil animal species in different soil types. Ecotoxicol. Environ. Saf. 33: 128-136. Neher, D. A. 1995. Biological diversity in soils of agricultural and natural ecosystems. Pages 55-72 dans R. K. Olson, C. A. Francis, and S. Kaffka, eds. Exploring the role of diversity in sustainable agriculture. ASA, Madison, WI.
Neher, D. A. and Campbell, C. L. 1994. Nematode communities and microbial biomass in soils with annual and perennial crops. Appl. Soil Ecol. 1: 17-28.

Neher, D. A., Peck, S. L., Rawlings, J. O. and Campbell, C. L. 1995. Measures of nematode community structure for an agroecosystem monitoring programm and sources of variability among and within agricultural fields. Plant Soil 170: 167-181.

Norton, R. A. 1994. Evolutionary aspects of oribatid mites life histories and consequences for the origin of the Astigmata. Pages 99-135 dans M. A. Houck, ed. Mites: ecological and evolutionary analyses of life-history patterns. Chapman and Hall, New York, NY. Petersen, H. 1995. Temporal and spatial dynamics of soil collembola during secondary succession in danish heatland. Acta Zool. Fenn. 196: 190-194.

Petersen, H. and Gjelstrup, P. 1995. Development of a semi-field method for evaluation of laboratory tests as compared to field conditions. Pages 67-142 dans H. Lokke, ed. Effects of pesticides on Meso- and Microfauna in soil. Danish Environmental Protection Agency, Copenhagen, Danemark

Popovici, I., Stan, G., Stefan, V., Tomescu, R., Dumea, A., Tarta, A. and Dan, F. 1977. The influence of atrazine on soil fauna. Pedobiologia 17: 209-215.

Ramade, F. 1992. Précis d'écotoxicologie. Collection d'Ecologie $\mathrm{n}^{\circ} 22$. Masson, Paris, France.

Sabatini, M. A., Pederzoli, A., Fratello, B. and Bertolani, R. 1979. Microarthropod communities in soil treated with atrazine. Boll. Zool. 46: 333-341.

Sarkar, K., Pramanik, R. and Joy, V. C. 1997. Sublethal toxic stress of heptachlor, a cyclodiene insecticide on saprotrophic soil microarthropods. J. Environ. Pollut. 4: 153-160.

Siedentop, S. 1995. A litter-bag test for the assessment of side effects of pesticides on soil mesofauna. Acta Zool. Fenn. 196: 357-360.

Stark, J. D., Tanigoshi, L., Bounfour, M. and Antonelli, A. 1997. Reproductive potential: its influence on the susceptibility of a species to pesticides. Ecotoxicol. Environ. Saf. 37: 273-279.

Toft, S. and Jensen, A. P. 1998. No negative sublethal effects of two insecticides on prey capture and development of a spider. Pestic. Sci. 52: 223-228.

Travé, J., André, H. M., Taberly, G. and Bernini, F. 1996. Les Acariens Oribates. Agar \& Sialf, Belgique.

Vreeken-Buijs, M. J., Hassink, J. and Brussaard, L. 1998. Relationships of soil microarthropod biomass with organic matter and pore size distribution in soils under different land use. Soil Biol. Biochem. 30: 97-106.

Wiles, J. A. and Frampton, G. K. 1996. A field bioassay approach to assess the toxicity of insecticide residues on soil to collembola. Pestic. Sci. 47: 273-285.

Wardle, D. A., Yeates, G. W., Watson, R. N. and Nicholson, K. S. 1995. The detritus food-web and the diversity of soil fauna as indicators of disturbance regimes in agro-ecosystems. Pages 35-43 dans H. P. Collins, G. P. Robertson, et M. J. Klug, eds. The significance and regulation of soil biodiversity. Kluwer Academic Publishers, Pays-Bas.

Zwart, K. B. and Brussaard, L. 1988. Soil fauna and cereal crops. Pages 139-168 dans L. G. Firbank, N. Carter, J. F. Darbyshire, et G. R. Potts, eds. The ecology of temperate cereal fields. Blackwell Scientific Publications, Oxford, RU. 
